\title{
REAL-TIME DATA DRIVEN ARTERIAL SIMULATION FOR PERFORMANCE MEASURES ESTIMATION
}

\author{
Dwayne Henclewood \\ Angshuman Guin \\ Randall Guensler \\ Michael Hunter
Civil and Environmental Engineering
Georgia Institute of Technology
Atlanta, GA 30332, USA

\author{
Richard Fujimoto \\ Computational Science and Engineering \\ Georgia Institute of Technology \\ Atlanta, GA 30332, USA
}

\begin{abstract}
Transportation professionals are increasingly exploring multi-pronged solutions to alleviate traffic congestion. Real-time information systems for travelers and facility managers are one approach that has been the focus of many recent efforts. Real-time performance information can facilitate more efficient roadway usage and operations. Toward this end, a dynamic data driven simulation based system for estimating and predicting performance measures along arterial streets in real-time is described that uses microscopic traffic simulations, driven by point sensor data. Current practices of real-time estimation of roadway performance measures are reviewed. The proposed real-time data driven arterial simulation methodology to estimate performance measures along arterials is presented as well as preliminary field results that provide evidence to validate this approach.
\end{abstract}

\section{INTRODUCTION}

Traffic congestion incurs a cost of an estimated eighty-seven billion dollars each year in the US. In 2007, Americans spent approximately four billion additional hours in traffic and purchased an estimated three billion gallons of additional gasoline due to congestion (Schrank and Lomax 2009). Addressing this high cost of congestion is a primary issue facing many of today's urban and suburban areas. However, as resources have become increasingly constrained, and right-of-way and construction costs have increased, transportation system managers and users have had to fundamentally shift away from new roadway capacity as the primary method for alleviating congestion.

This shift is reflected in the substantial efforts by governments, private industry, and the research community to develop and implement alternate means for alleviating congestion. As part of these broader efforts this paper will present a framework to provide the public and transportation facility managers with real-time and near-future arterial performance measures. An approach based on online data driven, microscopic traffic simulation utilizing point sensor data is used to provide these performance measures. This current and forecasted performance information will allow for greater efficiency in the use and operation of existing transportation facility capacity.

It is expected that this research will add to existing Advance Traffic Management System (ATMS) and Advance Traveler Information System (ATIS) efforts. Such systems have already been successfully utilized to reduce, or limit the increase in, freeway congestion in light of increasing travel demand. Many examples exist of system management and user services that have been developed to aid system managers in quickly identifying and addressing system breakdowns and provide travelers with current traffic conditions (RITA 2009; Google 2009; NAVTEQ 2009). However, most of these systems are focused on free- 


\section{Henclewood, Guin, Guensler, Fujimoto and Hunter}

way operations. The development of methods to accurately monitor real-time arterial traffic conditions and predict arterial congestion has received significantly less attention than the development of freeway methods.

In developing an approach to quantify real-time and predictive performance measures, particularly focusing on arterials, this paper will build upon an earlier feasibility study presented in (Henclewood, Hunter, and Fujimoto 2008). The proposed real time simulation based conceptual framework is presented along with descriptions of key aspects of the framework. Results of a field test of real-time simulation using a small scale implementation of the real-time performance measure portion of the framework are also presented. The field test provides evidence concerning the likelihood that a full-scale implementation of the framework will produce accurate estimates of performance measures. This paper concludes with a discussion of the limitations of this approach and areas of future work.

\section{RELATED WORK}

Arterial performance estimation presents many challenges. Interrupted traffic flow due to traffic signal timing and turn movements, lack of access control to prevent vehicles from entering via driveways, roadside traffic friction associated with parking activity, presence of urban buses and taxicabs, activity of pedestrians, and variability in local travel demand all contribute significantly to volume and speed variations along a corridor. Hence, it is not surprising that monitoring and predicting arterial congestion has proven to be much more difficult than that of freeways.

The development of models to estimate arterial performance measures began in the 1970s. These efforts focused on post evaluation of arterial performance using off-line estimation techniques. In 1977 P.G. Gipps provided one of the earliest models for estimating performance measures along arterials using a regression model that employed occupancy measurements and vehicle arrival times obtained from loop detectors to estimate arterial link travel times. Gipps noted that in order to improve the accuracy of his model, incorporating other parameters such as signal timing plans, number of lanes, and link lengths was needed (Gipps 1977). A number of researchers have used the Gipps model as a foundation to improve the estimation of arterial performance measures (Sisiopiku and Rouphail 1994; Gault and Taylor 1977).

Many of the earlier methods to estimate arterial performance are presented in comprehensive reviews such as those presented in (Sisiopiku and Rouphail 1994) and (Zhang and Kwon 1997). The former points out limitations of many existing methods; ranging from the lack of real-world validation results to the use of assumptions that may prevent the respective model from being implemented in the real-world (Sisiopi$\mathrm{ku}$ and Rouphail 1994). A number of more recent efforts have been devoted to addressing limitations and refocusing the assumptions of earlier models through the use detector data streams. For example, H. M. Zhang developed the Link-Journey-Speed (LJS) model that estimates the speed, and subsequently the travel time, along signalized arterials by incorporating volume and occupancy measurements obtained from loop detectors (Zhang 1999). In 2007, Liu and Ma presented a time-dependent model to estimate travel time along arterials using loop detector and signal status data (Liu and Ma 2007). Wang and Hobeika presented a modified HCM2000 model to estimate travel time along arterials using speed and volume data collected by upstream loop detectors (Wang and Hobeika 2007).

In contrast to post-determination, other efforts have attempted to provide real time arterial performance measures. Skabardonis and Geroliminis proposed an analytical model to estimate travel times along arterial streets in real-time. This model utilizes loop detector data, paired with signal timing data. Kinematic wave theory was then used to represent the spatial and temporal features of queues formed at signalized intersections. The travel time on an arterial link is calculated as the sum of the link free flow travel time and the delay experienced at the intersection (Skabardonis and Geroliminis 2005; Skabardonis and Geroliminis 2008). In (Kwong et al. 2009) a scheme for estimating the distribution of travel time on an arterial link is presented. The scheme employs the use of wireless sensors to acquire the magnetic signature of each vehicle. An upstream signature is matched with the signature from a downstream sensor to estimate the travel time of a particular vehicle. The authors also highlight that other performance meas- 


\section{Henclewood, Guin, Guensler, Fujimoto and Hunter}

ures such as the distribution of link volume, delay and queue length can be determined from this methodology (Kwong et al. 2009).

In Melbourne, Australia engineers investigated the feasibility of extracting arterial travel time measures in real-time from a small signalized arterial corridor using online data in conjunction with historical travel timereal-times. However, a potential drawback of this approach is the dependence on a particular control system and extensive detectorization (Luk, Karl, Su, and Bennett 2006). Another methodology (Wahle 2001) employs the use of cellular automaton microscopic traffic simulation software and approximately 750 inductive loop detectors located throughout Duisburg, Germany, to estimate roadway performance. A team of researchers from the University of Minnesota developed the SMART-SIGNAL system (Systematic Monitoring of Arterial Road Traffic and Signals) to estimate arterial performance measures in real-time (Liu, Ma, and Wu 2009). Integral to the functionality of this system is the collection of high-resolution event based traffic data primarily from signal controller cabinets. A field implantation of this system indicated that it is capable of producing accurate performance estimates in real-time, although difficulties involved with accessing event-based signal controller data directly from a roadside cabinet may limit widespread implementation. (Liu, Ma, and Wu 2009)

\section{METHODOLOGY}

The methodology employed in this effort uses point sensor traffic data to drive an online, microscopic traffic simulation in real time. Currently, point speed and volume data are utilized; however, the detectors are also capable of providing presence, occupancy, headway, and vehicle classification data. Given the real-time data streams and a representation of static conditions (number of lanes, allowed vehicle movements, lane lengths, etc.) as inputs; the traffic simulation package estimates current and near-future performance measures. The presented efforts use the VISSIM traffic simulator; however, the methodology could be applied to any microscopic simulation tool that can be adapted to accept real time point sensor data during run-time. The conceptual framework, along with details regarding critical framework components, is presented next.

\subsection{Conceptual Framework}

Figure 1 illustrates the conceptual framework for the developed real time, online, data-driven simulation system. The first step is to obtain real time traffic data from the roadway detectors. These data are transmitted to a data processing server where they are first subjected to quality control algorithms. The data processing server then sends the data to the simulation client where the current traffic state is estimated by utilizing the processed detector data to populate a simulation model of the area being studied. Once the traffic's current state is captured in the simulated environment, the model may be used to provide an estimate of future traffic conditions. For example, several other simulated instances of the traffic's current state may be generated faster than real-time, providing a collection of possible future traffic states. From these possible future states, the future state with the highest likelihood can be estimated by aggregating many possible future states, selecting a single state from possible states, combining selected predictions with historical data, or by using some other strategy. Both the probable future traffic state as well as the traffic's current state may then be transmitted to the various end users. The current results reported in this paper focus on the estimation of the current traffic state. However, it is noted that the general framework does incorporate near-term predictions. 


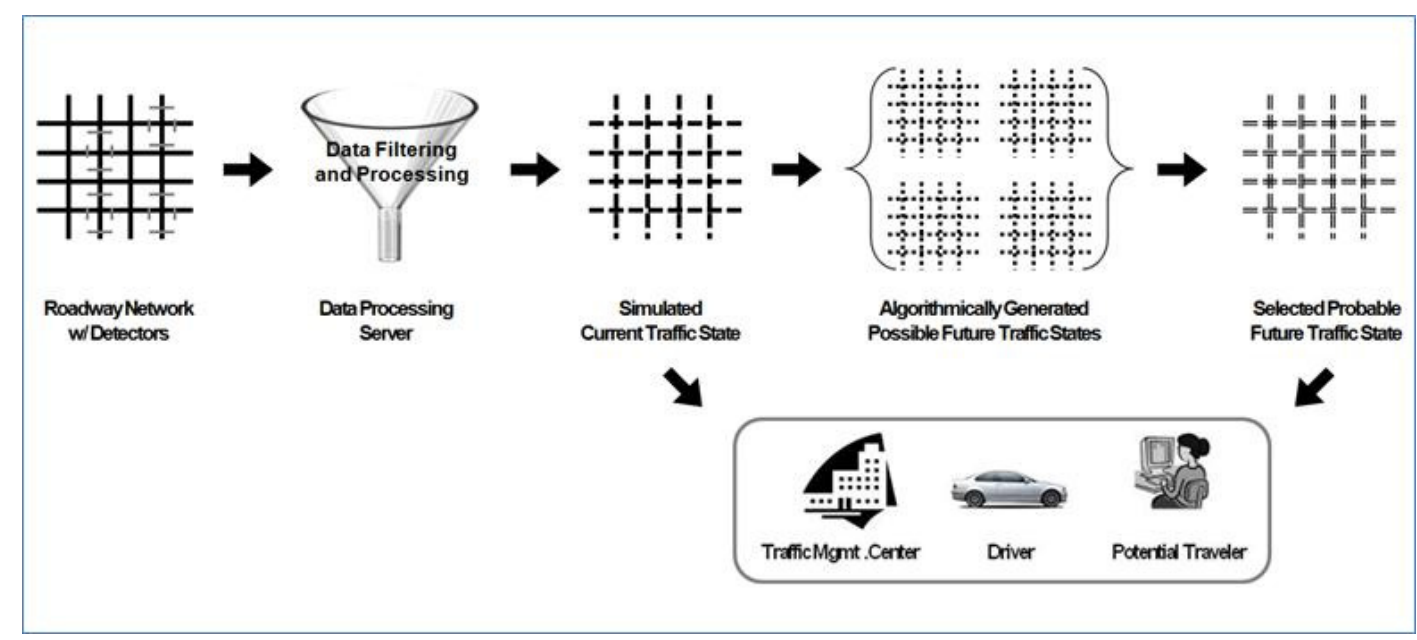

Figure 1: Conceptual framework for proposed methodology

\subsubsection{Roadway Network and Network Detectors}

Arterial networks are often comprised of streets with signalized and non-signalized intersections, where inductive loop detectors typically accompany each vehicle-actuated signalized intersection. The presence of the loop detectors, or any other form of point sensors that collect traffic data, is essential, as the data from such sensors drive the online simulation. It is assumed in this effort that the sensors can transmit their detector data in real time. While it is not common for today's roadway sensors to have real time transmission capabilities, such technology does exist and it is being increasingly adopted.

It is also assumed that the detector locations are known and may be mapped to the simulation environment and that the data being provided by point sensors include time stamped vehicle actuation and speed information. As previously mentioned, additional point sensor data streams commonly include lane occupancy, headway, and vehicle classification. Depending on the sensor technology used, some of these measurements may be drawn directly from the sensor while others may require additional processing of the raw data. While not currently used in this effort, it is anticipated that these data stream will be utilized in future efforts to further enhance the real-time simulation calibration.

\subsubsection{Data Processing and Communication}

The data that are sent by the various point sensors are processed to facilitate implementation in the simulation environment. The data processing unit's capabilities must account for the strengths and limitations of the different sensor technologies employed, such as inductive loops, pneumatic tubes, video detection system, microwave sensors, etc. In terms of strengths and weaknesses of the different technologies, the data processing unit will be able to, for instance, weigh the various input data from a video detection system on a cloudy, rainy day. This is due to that fact that these systems are known to underperform in such conditions given their reliance on being able to distinguish between a vehicle and the roadway surface.

The communication structure necessary for the framework implementation must satisfy three primary tasks: 1) manage the transmission of traffic data between the various point sensors and the data processing unit(s) via wired and/or wireless connection, 2) facilitate the communication between the data processing unit(s) and the various simulation instances, and 3) disseminate the current and most likely future traffic states. These communications/messaging tasks are primarily handled by software referred to as the Transportation Runtime Infrastructure (TRTI).

The TRTI is inspired by the High Level Architecture (HLA) (Symington, Morse, and Petty 2000) communication framework that manages the passing of information between simulation instances, re- 


\section{Henclewood, Guin, Guensler, Fujimoto and Hunter}

ferred to as federates. TRTI defines an application programming interface (API) based on a publish/subscribe model. This mode of operation allows clients (federates) to publish data to other federate(s) and receive data from the other federate(s) that have also subscribed to receive this information. The TRTI allows one or more federate to act as servers that orchestrate the sharing of information amongst the other federates. The TRTI framework supports both reliable and best effort communication. Another key feature of TRTI is that it allows users to manage, create, add or delete clients whenever there is a need to do so. For further details regarding the features of TRTI and its functionality see (Thiruvengadachari, Hunter, and Fujimoto, (2009); Thiruvengadachari, Hunter, and Fujimoto 2009).

\subsubsection{The Simulated Environment and Algorithm Development}

As stated earlier the microscopic traffic simulation used in the initial implementation of the framework is VISSIM (PTV 2010). VISSIM is a high resolution simulation program that is capable of modeling multimodal traffic flow. It is used primarily for studying traffic management policies and mechanisms under different traffic scenarios, and examining the effect(s) of various traffic phenomena, theories and technologies. In addition to VISSIM's ability to efficiently model and visualize traffic flow, it also provides a COM (Component Object Model) interface that allows VISSIM to run within other applications. The COM interface also provides users access to most VISSIM's objects during run time such that they may be created, manipulated or deleted to aid in the further examination of the traffic scenarios being studied. For additional information regarding VISSIM and the VISSIM COM interface see PTV (2009) and PTV (2010). A calibrated VISSIM model of the area being studied is essential to the proposed framework. A well calibrated model will help minimize potential discrepancies in the performance measures between the real-world and those of the simulation model. There may be several sources for such discrepancies, such as inaccuracies in the underlying vehicle behavior model, incorrect parameter settings, and errors in the data used to drive the simulation. Real-time calibration provisions will work best when making relatively small adjustments with the methodology, likely being most effective when starting with a wellcalibrated VISSIM model. Currently, real-time data streams are utilized to dynamically drive traffic volumes and desired vehicle speeds at the network boundaries, while internal network dynamic characteristics, such as turn movements and signal control, are based on historical data.

Future efforts will seek to develop a set of algorithms to undertake real time calibration of the global VISSIM model parameters and vehicle operations on internal network links. For instance, internal link speeds and densities may be adjusted by continuously monitoring and comparing link volume and detector occupancy across the real-world and the simulated environment. When, for example, the difference in these two set of values exceed a pre-set threshold, a calibration algorithm could be invoked that alters the necessary system parameters to tune the performance measures in the simulated environment to better reflect those of the real-world.

\subsubsection{Information Dissemination}

The last component of the methodology is the dissemination of the traffic information. The type and quantity of information that should be delivered to users depends on the user needs. For instance, drivers may only need the current and near-future travel time and/or travel speed along the links that make up their simulated or pre-selected path. On the other hand, a system manager may want the same information for a network of arterials, along with other performance measurements such as average headway, occupancy, density, and flow. One of the keys to delivering traffic information to the respective user will be ensuring that the data are in a readily digestible format (i.e. easily interpreted) so that users can make appropriate decisions. Web-based dissemination of aggregate data may be sufficient for drivers. However, transportation facility managers may need access to more detailed information, and possibly even raw data values. The system itself will need detailed data to adjust traffic system parameters such as signal timing information that are used by the models. 


\section{FIELD EXPERIMENT}

In prior work, our research team conducted a number of preliminary studies to determine the feasibility of the proposed framework. One of the first tasks was to develop a simulation environment that allows one to reflect arterial performance measures during real time runs. This was facilitated through the use of two simulation instances of a study corridor, with one instance representing a reference-world and the other, a model-world. The goal of this task was evaluate how well the model-world is able to replicate the performance measures of the real-world by extracting roadway data from the reference-world and using them as the driving input for the model-world. This extraction is currently handled by incorporating point sensor data within the traffic simulation package. The COM interface was used to modify VISSIM for this purpose. In essence, the COM modifications allow the simulation model to write output data for these embedded traffic sensors as the data are created, such that the external data file can be read and used by other programs (using the TRTI for communication between federates) while the simulation model is running. For a complete description of this feasibility test and its results see (Henclewood, Hunter, and Fujimoto 2008). Similar COM interface modifications were subsequently made so that boundary conditions, such as traffic flow volumes into a simulation link, and mid-block volumes and speeds could be delivered into the simulation from other simulation runs or from real-world, real time traffic monitors.

Given the feasibility of the proposed methodology in a simulated environment, a field test was developed to explore the methodology's robustness. The goal of the initial field test was, in part, to determine whether a VISSIM simulation instance could be driven by real time real-world detector data and produce performance measures that reflect those of the area being simulated. To conduct this experiment, the $5^{\text {th }}$ Street / Ferst Drive corridor in the midtown Atlanta area on the Georgia Tech campus was selected as the arterial to be studied (see Figure 2a). The researchers developed a VISSIM model for the test bed area, with boundary conditions driven by streaming detector data and with midblock detector inputs (Figure 2b). Each detector has a unique location ID and is capable of transmitting a time-stamped message that includes notations of a vehicle's presence, travel lane, and estimated speed. In addition, the corridor was outfitted with several temporary cameras located at the various intersections that record arterial operations during this experiment. The cameras also facilitate the extraction of performance measures, namely travel time, from the video data; which is then used in post-hoc analysis of the real-time simulation performance. Supplementing these data, two GPS equipped vehicles logged their location and speed data as they traverse the study corridor during the experiment. Figure $2 b$ shows the VISSIM representation of the test site and the locations of detectors and cameras along the $5^{\text {th }}$ Street NW and Ferst Drive NW corridor.

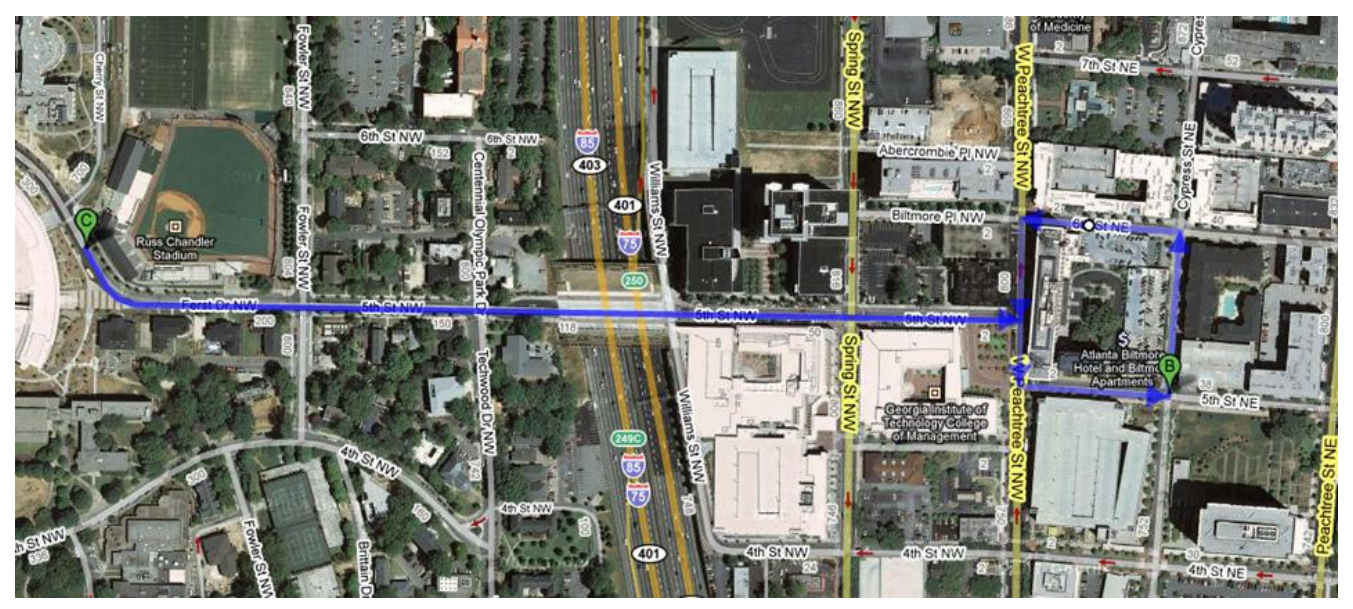

Figure 2a: Aerial of the $5^{\text {th }}$ Street NW/Ferst Drive NW Corridor, Atlanta GA (Google 2009) 


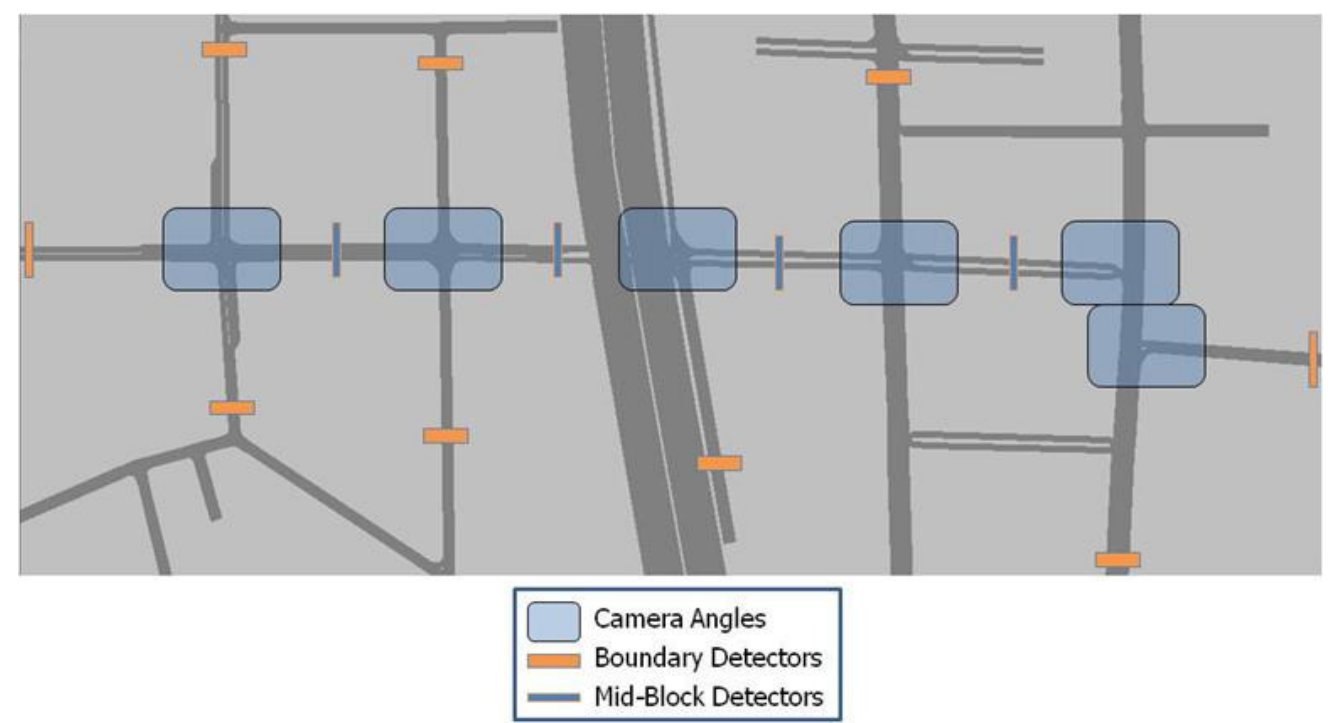

Figure 2b: VISSIM representation of the study corridor and the location of detectors and cameras

The experiment was conducted for 90-minutes, during a peak noontime period in the summer of 2009. The experiment involved detectors that transmitted data over Georgia Tech's wireless network to a central data processing server. A time stamped message was sent for each vehicle that crossed a detector. The time stamped data includes the link number and lane number of the reporting detector and the measured vehicle speed. The real time data stream was fed into the VISSIM simulation model of the area as it progressed in sync with wall clock time. Also during the experiment, the two instrumented vehicles made continuous round trips along the corridor while their GPS units recorded the vehicles' speed and latitude/longitude location every second. The cameras recorded vehicle activities at the six intersections in the corridor. At the end of the 90-minute test period the logged data was processed and various performance measures extracted for comparison with the simulation output.

\subsection{Results and Analysis}

The camera and video based travel time data were compared with that produced by the VISSIM model to determine how well the data-driven simulation was able to reflect real-world travel times. From this field experiment, two primary sets of travel times were obtained: eastbound (EB) and westbound (WB). Each set of travel times has an "actual" (real-world) and a VISSIM travel time component. To determine how well the proposed methodology estimates arterial performance measures in real-time, comparisons were based on which direction the vehicles are traveling and whether or not performance measures are actual or estimated by VISSIM.

The first step in comparing the travel time measurements was to create a scatter plot of the various data sets. See Figures $3 a$ and $3 b$. The primary goal of these scatter plots was to inspect and make preliminary inferences regarding how similar the data were within each set. When looking at the eastbound data set, Figure 3a, one can infer that the VISSIM travel times are similar to the actual travel times, with exceptions at the edges of the graphic where VISSIM travel times seem to be higher than the actual travel time. For the westbound data sets, Figure 3b, there is less similarity between the VISSIM and the actual travel times. From the westbound graphic the actual travel times appear systematically in the lower range of travel times output by VISSIM. 


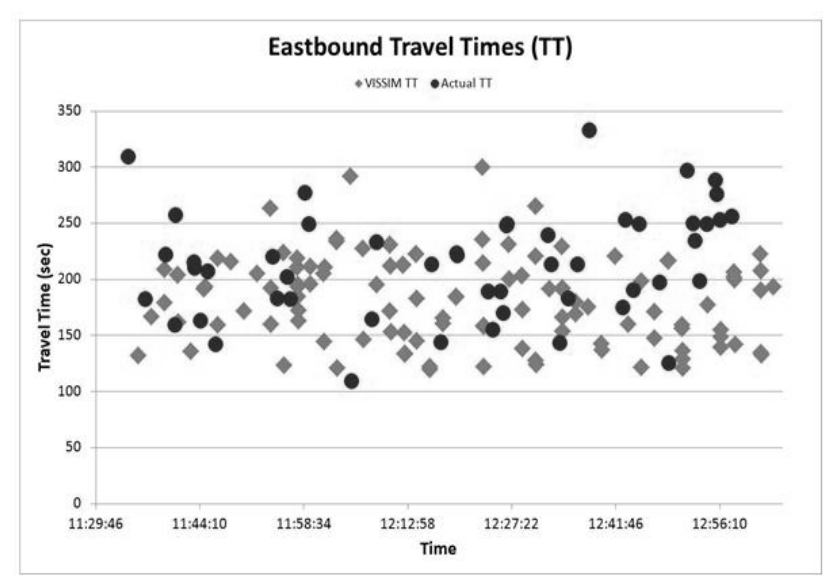

(a)

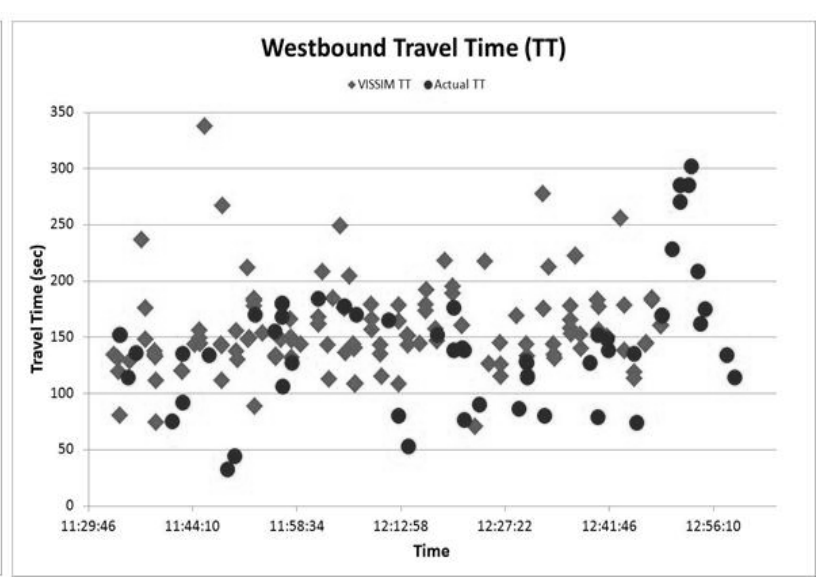

(b)

Figure $3 \mathrm{a}$ and $3 \mathrm{~b}$ : Eastbound and Westbound VISSIM Travel Times vs. Actual Travel Times from Instrumented Vehicles

The descriptive statistics were next examined (see Table 1). In the eastbound dataset, the VISSIM travel times have a mean of 183.1 seconds and a standard deviation of 39.3 seconds. And the actual travel times have a mean of 218.4 seconds and a standard deviation 50.1 seconds. Although these means are seemingly different, additional statistical tests were performed to assess this inference. It is interesting to note that the higher Eastbound actual travel time does not appear to be systematic but heavily influenced by the cluster of high values near the end of the run. An analysis of the data removing the last fifteen minutes reduces the difference in average travel time between the Eastbound simulated and field results by more than fifty percent. Potential reasons for this cluster will be discussed later in the section. As for the westbound direction, the mean and standard deviation of the VISSIM travel times are 157.5 seconds and 38.9 seconds respectively, while for the actual travel times these statistics are 113.4 seconds and 63.0 seconds respectively.

Table 1: Descriptive Statistics for Travel Times Eastbound and Westbound Directions

\begin{tabular}{ccccc}
\hline Statistic & \multicolumn{2}{c}{ Eastbound Travel Time } & \multicolumn{2}{c}{ Westbound Travel Time } \\
& VISSIM & Actual & VISSIM & Actual \\
\hline Mean & 183.1 & 218.4 & 157.5 & 113.4 \\
Standard Deviation & 39.3 & 50.1 & 38.9 & 63.0 \\
\hline
\end{tabular}

To further understand the data, a number of statistical tests were conducted. The main statistical test is aimed at determining whether the VISSIM and the actual travel times are statistically different for each direction as well as whether or not the each set of travel times are from the same distributions. First the distributions were tested for normality as this will influence the statistical test chosen. Lilliefors normality tests were conducted on all the travel time data sets. The results of the normality tests are presented in Table 2. From these results one is unable the reject the null hypothesis that the eastbound VISSIM and actual travel times are normally distributed. As a result, to determine if there are statistical differences between the VISSIM and the actual travel times, a parametric test may be more suitable for the eastbound data while a non-parametric test may be more suited for the westbound data. The parametric test chosen to evaluate the statistical difference between the eastbound data is a two sample t-test while the selected non-parametric test for the westbound data is the Wilcoxon Rank Sum test. For a sense of completion, these selected comparison tests will also be conducted on the travel time data that normality tests suggested they are not suitable for. In testing whether or not each dataset is from the same distribution a ChiSquare Test and a Kolmogorov-Smirnov test were conducted. The results from the comparison tests are also represented in Table 2. 
Table 2: Statistical Test Results

\begin{tabular}{|c|c|c|}
\hline Statistical Test & p-Value & Interpretations \\
\hline \multicolumn{3}{|l|}{ Normality Test } \\
\hline EB VISSIM & 0.3255 & Unable to reject normality assumption \\
\hline WB VISSIM & 0.0001 & Reject normality assumption \\
\hline EB Actual & 0.6760 & Unable to reject normality assumption \\
\hline WB Actual & 0.0088 & Reject normality assumption \\
\hline \multicolumn{3}{|l|}{2 Sample t-Test } \\
\hline 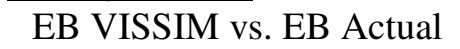 & 0.0001 & Reject equal mean assumption \\
\hline WB VISSIM vs. WB Actual & 0.1125 & Reject equal mean assumption \\
\hline \multicolumn{3}{|l|}{ Wilcoxon Sum Rank Test } \\
\hline EB VISSIM vs. EB Actual & 0.0001 & Reject equal median assumption \\
\hline WB VISSIM vs. WB Actual & 0.0408 & Reject equal median assumption \\
\hline \multicolumn{3}{|l|}{ Chi-Square Test } \\
\hline EB VISSIM \& EB Actual & 0.3654 & Unable to reject same distribution assumption \\
\hline WB VISSIM \& WB Actual & 0.1560 & Unable to reject same distribution assumption \\
\hline \multicolumn{3}{|l|}{ Kolmogorov-Smirnov Test } \\
\hline EB VISSIM \& EB Actual & 0.0016 & Reject same distribution assumption \\
\hline WB VISSIM \& WB Actual & 0.0235 & Reject same distribution assumption \\
\hline
\end{tabular}

From the above results, one can conclude that irrespective of the comparison test performed, there is a statistical difference between the VISSIM and the actual (mean / median) travel times, in both the eastbound and the westbound directions. However, it is again noted that if the last fifteen minutes of data were not included in the eastbound analysis the result is reversed, with the test failing to reject equal means. This further indicates an event specific issue rather that a systemic problem eastbound. In looking at the results of the similar distribution tests, one observes that the results may not be conclusive in suggesting that each dataset is from the same distribution. Given these outcomes, the research team reexamined the entire experimental setup to attempt to find reasons that could justify the difference between the estimated and actual performance measures. It was hoped that after uncovering the possible sources of discrepancies and developing means to address those sources that the above discrepancies would not only be reduced but possibly eliminated and the feasibility of the proposed methodology would be reestablished.

Upon examining the experimental setup, several areas are perceived to be likely sources of the discrepancies between the estimated and actual performance measures. These areas are generally related to model calibration and facility representation. In terms of model calibration, the parameters that reflect driver behavior were left unchanged in VISSIM, which is indicative of the fact that driver behavior in the VISSIM model may not be representative of the behavior along the study corridor. Accurately capturing driver behavior will improve VISSIM estimates of travel times. For example, aggressive or nonaggressive driving at various locales along the study corridor affects travel times. Additionally, turning movement distributions at the various intersections throughout the corridor were not accurately simulated. This is evidenced in the fact that the number of vehicles that traversed the travel time segment in VISSIM is approximately twice the number of vehicles that actually traversed the study corridor. With such a considerable difference in predicted vs. observed volumes on some links, it is possible that VISSIM is not accounting for congestion due to the higher volume level, which would likely lead to an increase in travel time. It is also noted that volume discrepancy could result from detector errors. It is possible the field detection system is missing vehicles and requires extensive recalibration. Finally, the Tech Trolley (an oncampus shuttle) was also not represented. By not capturing the Trolley behavior, VISSIM is not able to simulate the increase in travel time for other vehicles that the Trolley may inhibit as it traverses the corridor and makes its mandatory stops. 


\section{Henclewood, Guin, Guensler, Fujimoto and Hunter}

As for facility representation, there are two aspects of the study corridor that were not represented in the VISSIM model of the area. The first was the gradient of the roadway which goes uphill from west to east and the second being the pedestrian facilities, and pedestrians, along the corridor. If the corridor's gradient was represented accordingly, the westbound VISSIM travel times likely would be lower and be more representative of the actual travel times. Note, the inverse effect is not anticipated for the eastbound direction. It is believed that once driver behavior is calibrated accurately, simulated VISSIM vehicles will navigate the grade more similarly to the real-world drivers. As for pedestrian facilities and pedestrians, the probe vehicle drivers noted that there were times when allowable pedestrian movements significantly interfered with traffic flow. In particular, given the nature of college campuses there tends to be brief periods in which significant, short lived, increases in pedestrian activity occur. For example, left turning vehicles, yield to crossing pedestrians, and subsequently prevent vehicles from traversing the intersection from the same approach. Pedestrian activity is being explored as a likely cause of the short spike in eastbound real world travel time noted at the end of the experiment. It is anticipated that once the necessary adjustments are made to the VISSIM model, the estimated performance measures will more closely reflect those of the real world.

With the aforementioned possible sources of discrepancies in mind, the researchers believe that once the necessary changes are made to the VISSIM and the experimental setup, better estimates of arterial performance measures will be obtained. Despite these observed discrepancies between simulated and observed traffic behavior, the researchers were able to demonstrate the fundamentals of the proposed methodology in this field test. The microscopic traffic simulation model was able to be driven in real time by real-world data streams. The comparative analysis did provide some limited success, especially when looking at the eastbound scatter plot of travel times, in that there was some similarity in the performance measures from the real-world and those from the simulated environment. It is anticipated that once sources of discrepancies are addressed, then VISSIM will be able to produce better estimates of travel times.

\section{FUTURE DIRECTIONS}

Given the limited success from the above field test, there are a series of next steps that this research team has already begun to implement. The team is currently addressing a number of the previously identified internal modeling discrepancies (driver aggressiveness, etc.) and simultaneously developing additional tools and techniques aimed at allowing the methodology to more accurately estimate arterial performance measures in real-time. To further increase the accuracy of simulated performance measure estimates, three areas of concerns have been identified: input-volume accuracy, turning movement distribution, and signal synchronization.

Acquiring accurate volume estimates from point sensors is a perennial challenge faced by transportation practitioners. Although the technology varies from one detector to the next, accurate volumes tend to be difficult to obtain irrespective of technology. At this moment, the point sensor technologies that this effort is considering implementing include portable inductive loops and a video detection system. To combat the inevitable inaccuracies that are associated with these detection technologies, the research team will develop a corresponding algorithm to correct volume estimates based on a detailed analyses to understand the inaccuracies and how to best correct them.

Similar to the importance of acquiring accurate entering volumes to the network being studied, accurate estimates of turning movement proportions are also needed to provide correct, real time estimates and prediction of performance measures. The study of estimating turning movement proportions has been likened to the development of models that estimate dynamic origin to destination flows in a small network (Liu, Ma, and Wu 2009). As a result, works that present models for estimating turning movement proportions are abundant. Chang and Tao 1998 and Liu, Ma, and Wu 2009 presented a summary of some of the more notable works in turning movement proportion estimation. Also included in (Liu, Ma, and Wu 2009) is the authors' own simple model of determining the proportion of drivers that exercise the various 


\section{Henclewood, Guin, Guensler, Fujimoto and Hunter}

turning options at an intersection. This research project will build on the work by others in this area and hopes to develop a model that will facilitate greater accuracy in turning movement proportions.

Given the importance of traffic signal operations in determining performance measures along arterials, it is also necessary for a signal's state in the field to be synchronized with that which is in the simulated environment. For instance, if the simulation timing plan is out of sync by 15 -seconds, some vehicles that are predicted to stop at the signal would actually be passing through the signal on a green light in the real world. To synchronize traffic signal operations within the simulation and real-world environments, the research will build upon previous research efforts. The team anticipates that improved traffic signal synchronization between the environments will also improve comparative results. Once the relationships between comparative results and signal timing synchronization are better understood, the team will develop new methods to correct simulation outputs or re-synchronize signal timing plans in the simulation environment to better reflect real time, real-world conditions. For example, various field measurements such as detected lane occupancy and vehicle speed at an intersection might be used to synchronize signal timing in the simulated environment with those in the field.

\section{CONCLUSION}

The above sections presented the development of a dynamic data-driven simulation based system for estimating and predicting performance measures along arterial streets, in real time, through the use of microscopic traffic simulation driven by point sensor data. Upon reviewing the state-of-the-art practices for estimating arterial performance measures in real time, this effort presented a conceptual framework that builds on the foundation early efforts. Additionally, this effort is seeking to make use of available technology to increase the feasibility and accuracy with which arterial performance measures are estimated. The results of a field test, which stemmed from the conceptual framework, were also presented. Although the comparison of VISSIM and actual travel times were statistically different, visual inspection of the eastbound travel times did provide some encouragement that the currently purposed methodology is feasible. Additionally, when one examines the current experimental set up and various parameters, especially as it relates to the VISSIM model, it can be concluded that after proper calibration of the model and accurate representation of the various features of the study corridor that the methodology will likely estimate arterial performance measures much more accurately than observed in this initial field test.

\section{REFERENCES}

Chang, G., and X. Tao. 1998. Estimation of Time-Dependent Turning Fractions at Signalized Intersections. Transportation Research Record, 1644(1), 142-149.

Gault, H. E., and I. G. Taylor. 1977. The Use of Vehicle Detector to Assess Delay in Computer Controlled Area Traffic Control. Newcastle.

Gipps, P. 1977. The Estimation of a Measure of Vehicle Delay from Detector Output. Newcastle.

Google. 2009. Google Maps. Web Page. Retrieved from http://maps.google.com/.

Henclewood, D., Hunter, M., and R. Fujimoto. 2008. Proposed Methodology for a Data-Driven Simulation for Estimating Performance Measures Along Signalized Arterials in Real-Time. In Proceedings of the 2008 Winter Simulation Conference, eds. S. J. Mason, R. R. Hill, L. Mönch, O. Rose, T. Jefferson, J. W. Fowler. Piscataway, New Jersey: Institute of Electrical and Electronics Engineers, Inc.

Liu, H. X., W. X. Ma, and H. H. Wu. 2009. Development of a Real-Time Arterial Performance Monitoring System Using Traffic Data Available from Existing Signal Systems. Queue. Minneapolis.

Kwong, K., R. Kavaler, R. Rajagopal, S. Networks, P. Varaiya, and C. Sciences. 2009. A practical scheme for arterial travel time estimation based on vehicle re-identification using wireless sensors. In TRB 2009 Annual Meeting (CD-ROM) (p. 15). Washinton DC: National Academies. 
Liu, H. X., and X. W. Ma. 2007. Time-dependent travel time estimation model for signalized arterial network. In 86th Transportation Research Board Annual Meeting 2007 (Vol. 1, p. 21). Washinton DC: National Academies.

Luk, J., C. Karl, M. Su, and P. Bennett. 2006. Real-time estimation of travel times on arterial roads in melbourne. In 22nd ARRB Conference - Research into Practice (p. 14). Canberra Australia: ARRB Group Ltd and Authors.

NAVTEQ. 2009. NAVTEQ Traffic.Com. Web Page. Retrieved from http://www.traffic.com/.

PTV. 2009. VISSIM 5.10-06 COM Interface Manual. Interface. Karlsruhe, Germany.

PTV. 2010. PTV America - VISSIM

RITA. 2009. Intelligent Transportation Systems. Web Page.

Schrank, D., and T. Lomax. 2009. Urban Mobility Report 2009. Report for the Texas Transportation Institute, (July).

Sisiopiku, V., and N. Rouphail. 1994. Toward the use of Detector Output for Arterial Link Travel Time Estimation: A Literature Review. Transportation Research Record, 2(1457), 158-165. Transportation Research Board.

Skabardonis, A., and N. Geroliminis. 2005. Real-time estimation of travel times on signalized arterials. In Proceedings of the 16th International Symposium on Transportation and Traffic Theory (pp. 1-21).

Skabardonis, A., and N. Geroliminis. 2008. Real-Time Monitoring and Control on Signalized Arterials. Journal of Intelligent Transportation Systems, 12(2), 64-74.

Symington, S., K. Morse, and M. Petty. 2000. IEEE Standard for Modeling and Simulation (MlandS)

High Level Architecture (HLA)-Framework and Rules (IEEE Std 1516-2000). Institute of Electrical

and Electronics. Institute of Electrical and Electronics Engineers, Inc., 2000.

Thiruvengadachari, P., M. Hunter, and R. Fujimoto. 2009. TRTI - Overview. Georgia Institute of Technology, Atlanta GA.

Thiruvengadachari, P., M. Hunter, and R. Fujimoto. 2009. TRTI : Traffic Runtime Infrastructure for Adhoc Distributed Simulations (p. 6). Georgia Institute of Technology, Atlanta GA.

Wahle, J. 2001. A cellular automaton traffic flow model for online simulation of traffic. Parallel Computing, 27(5), 719-735.

Wang, H., and A. Hobeika. 2007. Travel Time Estimation on Arterial Streets. Review Literature And Arts Of The Americas. Blacksburg, VA.

Zhang, H. 1999. Link-Journey-Speed Model for Arterial Traffic. Transportation Research Record, 1676(1), 109-115.

Zhang, H., and E. Kwon. 1997. Travel Time Estimation on Urban Arterials Using Loop Detector Data. Iowa City.

\section{AUTHOR BIOGRAPHIES}

DWAYNE HENCLEWOOD is a Ph.D. Candidate at the Georgia Institute of Technology. His current research interest is in arterial performance evaluation and microscopic traffic simulation. He holds a M.S. in Civil Engineering from the University of Massachusetts Amherst. His email address is <dahenclelgatech.edu> .

ANGSHUMAN GUIN is a Research Engineer in the School of Civil and Environmental Department at the Georgia Institute of Technology. His research interests include intelligent transportation systems, freeway traffic operations, transportation safety and traffic simulation. His e-mail address is $<$ angshuman.guin@ce.gatech.edu> .

RANDALL GUENSLER is a Professor in the School of Civil and Environmental Department at the Georgia Institute of Technology. His areas of interest are transportation planning, vehicle activity monitoring, air quality modeling, environmental impact assessment, and environmental policy analysis. He 
earned his Ph.D. at the University of California at Davis. His e-mail address is <randall.guenslerece.gatech.edu>.

MICHAEL HUNTER is an Associate Professor in the School of Civil and Environmental Department at the Georgia Institute of Technology. His primary areas of interest are traffic safety, operational performance and control, and simulation. He earned his Ph.D. at the University of Texas at Austin in August 2003. His e-mail address is <michael. hunter@ce. gatech. edu>.

RICHARD FUJIMOTO is a Regents' Professor and Chair of the Computational Science and Engineering Division at the Georgia Institute of Technology. He received his M.S. and Ph.D. degrees from the University of California (Berkeley) in 1980 and 1983 respectively. Among his past activities he lead the definition of the time management services for the DoD High Level Architecture (HLA), and has also been a member of the steering committee for the Workshop on Parallel and Distributed Simulation, (PADS) since 1990. His email address is <fujimo-to@cc.gatech.edu>. 